\title{
THE ELASTIC STRAIN RATIO, THE LÜDERS STRAIN RATIO AND THE EVOLUTION OF $r$ - VALUE DURING TENSILE DEFORMATION
}

\author{
DOMINIQUE DANIEL and J. J. JONAS \\ Department of Metallurgical Engineering, McGill University, 3450 University St, \\ Montreal, Quebec, H3A 2A7, Canada
}

\section{J. BUSSIÈRE}

National Research Council Canada, Industrial Materials Research Institute, Boucherville, Quebec, J4B 6Y4, Canada

(Received September 17, 1991)

The elastic counterpart of the plastic strain ratio is derived from ultrasonic data measured on twenty commercial deep drawing steels. It is shown that the observed variations in plastic $r$ are related to the evolution of texture, and are not affected either by the elastic range of deformation or by the propagation of Lüders bands. Further quantitative analysis suggests that the elastic strain ratio, determined non-destructively, can be used to predict plastic $r$-values by means of an empirical relationship.

KEY WORDS Texture, ODF coefficients, plastic anisotropy, elastic anisotropy, $r$-value, Lüders deformation, cold rolled steel sheet.

\section{INTRODUCTION}

The plastic strain ratio or Lankford parameter (Lankford et al., 1950) is well understood physically as a measure of the capacity to resist thinning. It is generally referred to as the $r$-value:

$$
r=\frac{\varepsilon_{22}}{\varepsilon_{33}}=\frac{\ln w_{0} / w_{f}}{\ln t_{0} / t_{f}}
$$

where $\varepsilon_{22}$ and $\varepsilon_{33}$ are the true strains in the width and thickness directions defined by the initial and final widths and thicknesses $w_{0}, w_{f}, t_{0}$ and $t_{f}$. Because of the orthorhombic symmetry of rolled sheet, it need only be measured between the rolling $(0 \mathrm{deg})$ and transverse $(90 \mathrm{deg})$ directions. In practice, the average $\bar{r}$ is used to characterize a material:

$$
\bar{r}=\frac{1}{4}\left(r_{0}+2 r_{45}+r_{90}\right)
$$

Whiteley et al. (1961) showed that the higher the $\bar{r}$, the deeper the draw. The parameter $\Delta r$ is used in turn to quantify the planar anisotropy:

$$
\Delta r=\frac{1}{2}\left(r_{0}-2 r_{45}+r_{90}\right)
$$

which is related to the tendency to form four-fold ears. 
One of the problems involved in $r$-value measurement is that it is frequently observed to vary with strain (Arthey and Hutchinson, 1981; Welch et al., 1983; Liu, 1983, Liu and Johnson 1985; Hu, 1983; Lake et al., 1988). The rate of change of $r$-value is particularly rapid in the early stages of deformation (Daniel, 1990; Daniel and Jonas, 1990), leading to the possibility that elastic phenomena, including the elasto-plastic transition, are involved. The present work was undertaken with the aim of determining the extent to which elastic $r$-values, which generally differ from the plastic ones, play a role in this variation of $r$.

\section{ELASTIC STRAIN RATIO}

The elastic $r, r_{\mathrm{el}}$, can be calculated from the relation between the state of stress $\tilde{\sigma}$ and the resulting state of strain $\tilde{\varepsilon}$ (Hooke's law), as expressed by:

$$
\varepsilon_{i j}=s_{i j k l} \sigma_{k l}
$$

where $s_{i j k l}$ are the components of the fourth order elastic compliance tensors. In uniaxial tension along the 1 direction ( 2 is the width direction), the elastic strain ratio is therefore given by:

$$
r_{\mathrm{el}}=\frac{\varepsilon_{w}}{\varepsilon_{t}}=\frac{s_{1122}}{s_{1133}}
$$

These matrices can be transformed from the rolling frame to the tensile reference frame (inclined at an angle $\theta$ to the rolling direction) using the tensor transformation rule:

$$
s_{i j k l}^{\prime}=a_{i m} a_{j n} a_{k o} a_{l p} s_{m n o p}
$$

where the prime indicates the value of the constant in the tensile system, the $a_{i m}$ are the direction cosines relating the rolling and tensile coordinate axes, and the products are summed over all the mnop indices of the tensor. For a given $\theta$, $r_{\mathrm{el}}(\theta)$ is defined by:

$$
r_{\mathrm{el}}(\theta)=\frac{s_{1122}^{\prime}}{s_{1133}^{\prime}}=\frac{\cos ^{2} \theta \sin ^{2} \theta\left(s_{1111}+s_{2222}-4 s_{1212}\right)+\left(\cos ^{4} \theta+\sin ^{4} \theta\right) s_{1122}}{\cos ^{2} \theta s_{1133}+\sin ^{2} \theta s_{2233}}
$$

It should be noted that Poisson's ratio $v_{12}\left(=-\varepsilon_{22} / \varepsilon_{11}\right)$ is the elastic counterpart of the plastic contraction ratio $q$. These are the quantities measured in practice since the thickness strain $\varepsilon_{33}$ cannot be accurately determined on thin sheets. The relations between $r_{\mathrm{el}}$ and $v_{12}$, and $r_{\mathrm{pl}}$ and $q$ can be written as:

$$
r_{\mathrm{el}}=\frac{v_{12}}{1-v_{12}} \quad \text { and } \quad r_{\mathrm{pl}}=\frac{q}{1-q}
$$

where $r_{\mathrm{pl}}$ is the plastic strain ratio. Since $v_{12}$ in steels falls in the range $0.2<v_{12}<0.7, r_{\text {el }}$ can be expected to vary from about 0.25 to 2 . The latter is therefore smaller than $r_{\mathrm{pl}}$ (except when $r_{\mathrm{pl}}$ takes values between 0 and 1.5 , as it is shown later). As a result, $r$ generally increases as the material passes through the elastic-to-plastic transition. 


\section{PROBLEMS ASSOCIATED WITH THE EXPERIMENTAL DETERMINATION OF THE ELASTIC AND PLASTIC STRAIN RATIOS}

\section{a. Derivation of $r_{\mathrm{el}}$ from ultrasonic measurements}

The deformations associated with the elastic straining of a sample are so small that they cannot readily be measured directly. However, the elastic constants of the sheet can be derived nondestructively from measurements of the ultrasonic velocities (Sayers, 1982). The anisotropies of the longitudinal and shear sound velocities propagating in the rolling plane lead to a partial description of the polycrystal stiffness tensor $c_{i j k l}$ (Thompson et al., 1987, 1989; Hirao et al., 1988, 1989). More precisely, these ultrasonic data lead to only three independent elastic parameters, expressed linearly in terms of $c_{1111}, c_{2222}, c_{1122}$ and $c_{1212}$ (Sakata et al., 1990; Daniel, 1990; Daniel et al., 1991). A polycrystal elasticity model, such as the 2nd order Hill approximation (Bunge, 1974) is then needed in order to determine the entire stiffness tensor $c_{i j k l}$, from which the compliance tensor $s_{i j k l}$ can be obtained by matrix inversion. These operations lead to $r_{\mathrm{el}}$ according to Eq. (7).

\section{b. Direct measurement of $r_{\mathrm{pl}}$}

According to ASTM standard E517-81 pertaining to the plastic strain ratio, the width and length changes are measured after elongations of 15 to $20 \%$. The thickness strain is then calculated by assuming volume constancy. This requires that the errors in the length and width measurements before and after stretching not exceed about $10 \mu \mathrm{m}$ for the width and 25 to $50 \mu \mathrm{m}$ for the length, depending on the gage length. As a result of these stringent requirements, the quality of $r$-value measurements depends sensitively on the accuracy of the measuring technique and on the skill of the investigator.

The physical significance of the conventional definition (i.e. the accumulated strain ratio $\varepsilon_{w} / \varepsilon_{t}$ at $15 \%$ elongation) has, however, been questioned because of the evolution of the plastic anisotropy during straining. It has been pointed out that, both in mathematical modeling as well as for the characterization of material drawability, $r$ should be defined in terms of the instantaneous ratio $d \varepsilon_{w} / d \varepsilon_{t}$ at zero strain (Welch et al., 1983). This was suggested earlier by Hill (1948), but neglected because of the practical problems associated with carrying out experiments at such small strains. Measurement of the desirable instantaneous strain ratio requires complex equipment and involves a continuous record of the length and width changes during straining for later back extrapolation to zero strain. The need for accurate studies of plastic anisotropy has led to intensive research on the evolution of the strain state in tension (Liu, 1983; Liu and Johnson, 1985; Lake et al., 1988). This type of investigation has shown that, for a wide range of materials, the relation between the width and length strains can be considered as linear to a first approximation.

From strain data, such as those obtained in this study, two different definitions of $r$-value can be used. The first is the conventional one, consisting of the ratio of the accumulated width to thickness strain at a specified elongation, usually $15 \%$ $\left(\varepsilon_{1}=0.14\right)$. The second, called the regression $r$ in the present work, uses the slope of the linear relation $-\varepsilon_{w}=a+b \varepsilon_{1}$ obtained from pairs of width and length 

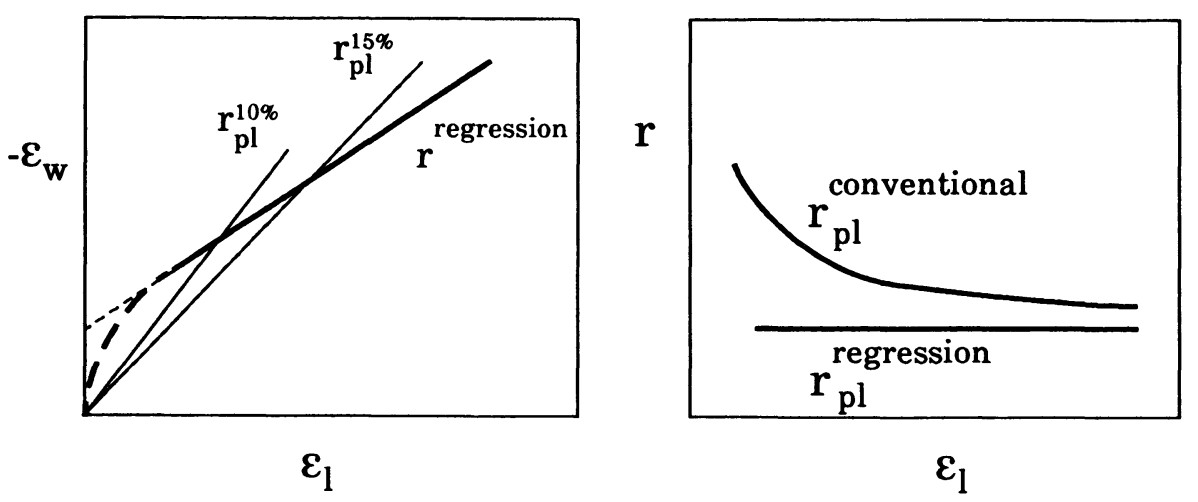

Figure 1 Schematic illustration of the definitions and strain dependences of the conventional and regression $r$-values (case of a positive intercept $a$ ).

strains measured during the course of (relatively) uniform elongation. These definitions are expressed, respectively, by the following equations:

$$
r_{\mathrm{pl}}^{\text {conventional }}=\frac{-\varepsilon_{w}}{\varepsilon_{l}+\varepsilon_{w}}
$$

and

$$
r_{\mathrm{pl}}^{\text {regression }}=\frac{b}{1-b}
$$

The major distinction between these two parameters is their different dependences on the strain. The conventional $r$ varies with elongation, at a rate that depends on the value of $a$. By contrast, the regression $r$ is constant by definition, which makes it attractive for characterizing the drawability. Figure 1 illustrates the differences between these two definitions.

The angular dependence of $r_{\mathrm{pl}}$ on $\theta$ (inclination to the rolling direction) can also be predicted from texture data (ODF coefficients). It was shown in earlier publications (Daniel, 1990; Daniel and Jonas, 1990; Daniel et al., 1991), that calculations using the $\mathrm{RC}_{3}$ relaxed constraint model are in good agreement with experimental measurements when appropriate values are selected for the critical resolved shear stress ratios for each steel.

\section{EXPERIMENTAL WORK}

\section{a. Materials}

Twenty samples of low carbon steel sheets were collected from various producers in Canada, United States and Japan. These were divided into five types: (1) batch-annealed Al-killed drawing quality (AKDQ) steels, (2) commercial-grade rimmed steels (denoted as RIM in the present work), high-strength low-alloy (HSLA) steels, and (4) and (5) two types of interstitial-free (IF) extra-low-carbon steel. The latter distinction was made because of the different textures observed (in terms of the $\{554\}\langle 225\rangle$ component (Daniel, 1990; Daniel and Jonas 1990), 
which induced different plastic anisotropies. In the first type of interstitial-free steel (IF1), the $\bar{r}$-values fell in the range from 1.5 to 1.9 , and the $\Delta r$ 's of 0.2 to 0.4 were below those of the AKDQ steels in the same $\bar{r}$ range. In the second type (IF2), the $\bar{r}$-values were above 2.0 , and the $\Delta r$ 's were still smaller, ranging from -0.2 to 0.2 .

\section{b. $X$-ray and acoustoelastic texture analyses}

The texture of each steel was analyzed by means of the X-ray reflection technique on a Siemens' goniometer system, consisting of a Digital Equipment PDP-11/73 controlled vertical diffractometer (D-500), attached to the theta (i.e. the Bragg angle $\theta$ ) circle of the Huber Eulerian cradle. Standard numerical methods (Bunge, 1982) provided the ODF coefficients from the incomplete pole figure data up to $l_{\max }=22$.

The present elastic measurements were carried out using electro-magnetic acoustic transducers, referred to as EMAT's. Two types of guided plate wave modes were employed: the lowest order symmetrical Lamb $\left(S_{0}\right)$ and the shear horizontal $\left(\mathrm{SH}_{0}\right)$ modes. The Lamb waves consist of a pair of transverse waves superimposed on a pair of longitudinal waves. Their direction of propagation lies in the plane parallel to the surface of the plate. For a given plate thickness, the wave velocity is a strong function of frequency, so that measurements are usually made in a frequency range where this dependence is weak. For the fundamental $\mathrm{S}_{0}$ mode, this range corresponds to frequencies far below the cutoff frequencies of the higher modes and the associated vibrations occur approximately parallel to the propagation direction. Nevertheless, there is a small lateral motion due to the plane stress boundary condition because the wavelength is long but not infinitely so (zero frequency).

The $\mathrm{S}_{0}$ and $\mathrm{SH}_{0}$ mode velocities were measured along three directions of propagation $\left(0^{\circ}, 45^{\circ}\right.$ and $90^{\circ}$ from RD) using Magnasonics EMAT's at a nominal frequency of $500 \mathrm{kHz}$. The $S_{0}$ mode velocities were extrapolated to zero frequency to correct for the dispersion effect associated with finite plate thickness (Daniel, 1990). This correction affects the measured values by $0.5 \%$, which is more significant than in the case of the resonant frequencies measured by the Modul-r tester. This is because the correction term is inversely proportional to the square of the wavelength, which is around $20 \mathrm{~cm}$ in the Modul-r case and $1 \mathrm{~cm}$ in the EMAT method. Due to the sheet symmetry, the $\mathrm{SH}_{0}$ mode velocities at $0^{\circ}$ and $90^{\circ}$ are equal. The 4th and 6th order texture coefficients were derived from the ultrasonic data by the method of Sakata et al. (1990), and used for calculation of the elastic and plastic $r$-values.

\section{c. Tensile testing}

To avoid the technical problems associated with the double extensometer technique, a time consuming but easier method than the interrupted test procedure was devised. Tensile tests were carried out on specimens cut from sheets at several orientations with respect to the rolling direction, i.e. every $15^{\circ}$, $22.5^{\circ}$ or $45^{\circ}$. For each orientation, two standard, parallel-sided, $200 \mathrm{~mm} \times 20 \mathrm{~mm}$ strip specimens (type B of ASTM 517-81) were first prepared. However, in order to be able to evaluate $\boldsymbol{r}$ at $\mathbf{1 0}$ to 15 strain levels by means of only one or two tests, 
the specimens were tapered from 20 to $19.3 \mathrm{~mm}$ within the gage length, following the suggestion of Liu (1983).

In the present work, a grid of $4 \mathrm{~mm}$ by $4 \mathrm{~mm}$ squares was scribed, using a laser beam technique, which led to a line width of about $50 \mu \mathrm{m}$. One of each pair of specimens was first stretched to $5 \%$ elongation and then to $10 \%$ at a strain rate of $2 \times 10^{-3} \mathrm{~s}^{-1}$; the second sample was stretched to $20 \%$ in a single test. The elongations in the first sample ranged from 5 to $12 \%$ along the center of the gage length after the second test. The strains induced by the taper ranged from 15 to $25 \%$ along the second sample. Grid measurement was carried out at seven locations along the gage length by means of a toolmaker's microscope, which ensured an absolute accuracy of $2.5 \mu \mathrm{m}$ in the readings. Due to the line thickness, the widths and lengths were determined with an accuracy of $20 \mu \mathrm{m}(\sim 0.2 \%$ error). The grid deformation was measured on $3 \times 3$ or $3 \times 2$ unit rectangles located along the center lines of the specimens.

\section{RESULTS AND DISCUSSION}

\section{a. Evolution of $r_{\mathrm{pl}}$ during tensile testing}

For each sample direction, an approximately linear relation between $\varepsilon_{w}$ and $\varepsilon_{1}$ was observed in the range of uniform deformation, as reported by Liu (1983) and Liu and Johnson (1985) for low carbon steels. Typical results for an IF1, an AKDQ and a RIM steel are presented in Figure 2. An interesting difference between the IF and other steels lies in the homogeneous yielding behaviour of the former grade, which affects the evolution of $\boldsymbol{r}$-value during straining, as described by Arthey and Hutchinson (1981) and Lake et al. (1988). As outlined by Lake et al. (1988), the absolute value of the intercept $a$ of the linear regression is higher for steels which undergo inhomogeneous yielding (Figure $2 b$ and $2 c)(|a| \approx 0.009)$ than for the IF steels (Figure $2 a)(|a| \approx 0.0015)$. Also, $a$ was found to be generally positive, although sometimes it was negative.

Although this value is subject to experimental error (Liu and Johnson, 1985; Lake et al., 1988), the large number of measurements carried out in the present work indicate that the $a$-value is close to zero for steels which yield homogeneously and non-zero for the others. In the latter case, high values of $|a|(>0.005)$ were found in the RIM and HSLA samples displaying high yield point elongations $(\sim 4-5 \%)$. The data for these specimens show larger deviations from linearity (see Figure 2c), which add some uncertainty to the $a$-values. Moreover these steels display earlier plastic instability than the AKDQ and IF grades. These two factors reduce the range of uniform deformation used for the regression and induce local inhomogeneities, which can also affect the determination of the conventional ASTM $r$-value.

To avoid the difficulties associated with the transition to homogeneous deformation, the regression proposed by Liu (1983) seems to be an appropriate technique for calculating the average strain ratio during deformation after propagation of the band. Furthermore, the number of points obtained at increasing strains improves the reliability of the estimated value. These statistical regression techniques lead to a $95 \%$ confidence interval for $b$, which corresponds to an error in $r$ of \pm 0.04 to \pm 0.08 (for steels displaying high yield point 
a) IF1

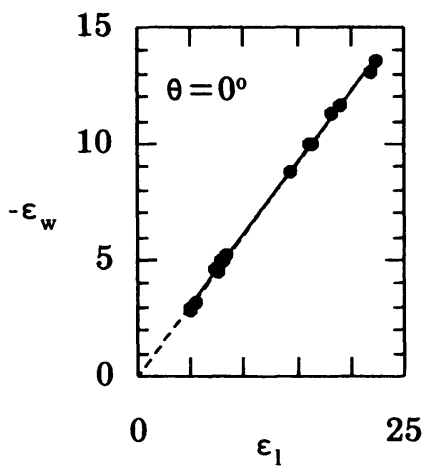

b) AKDQ

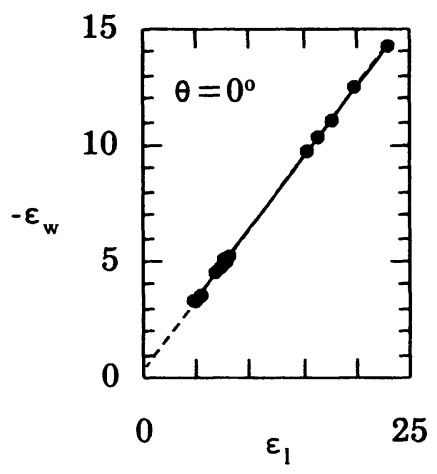

c) RIM

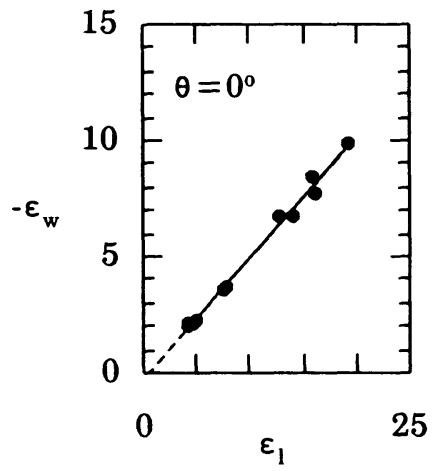

15

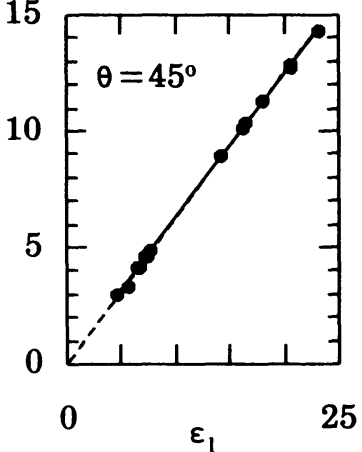

25
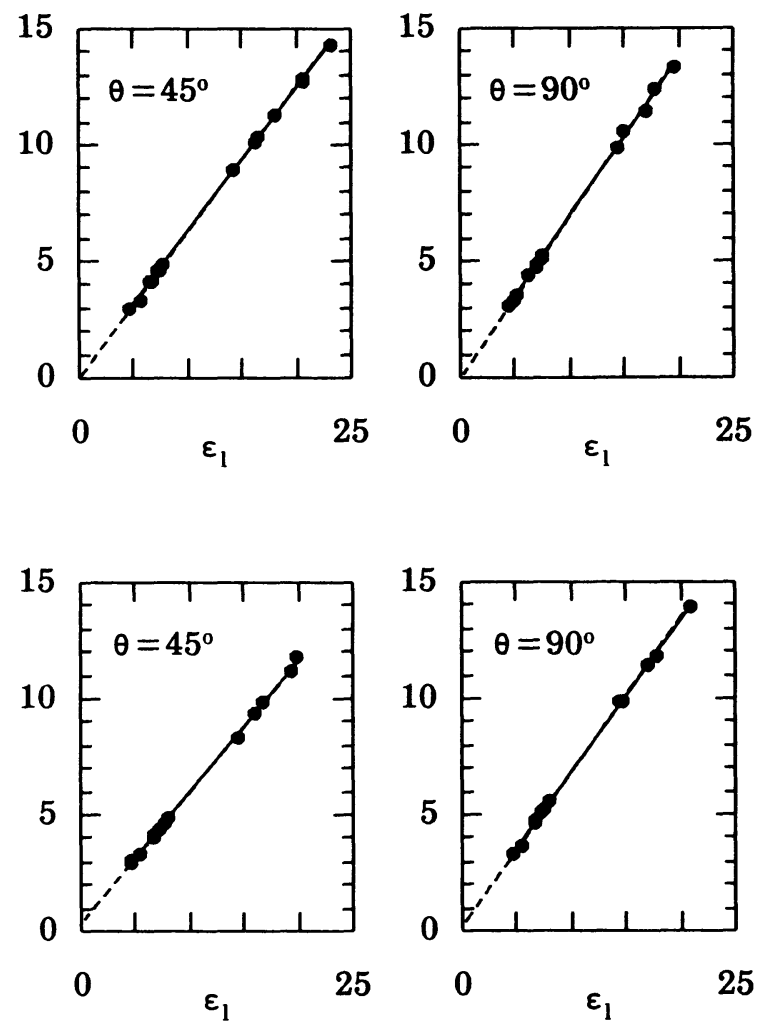

25
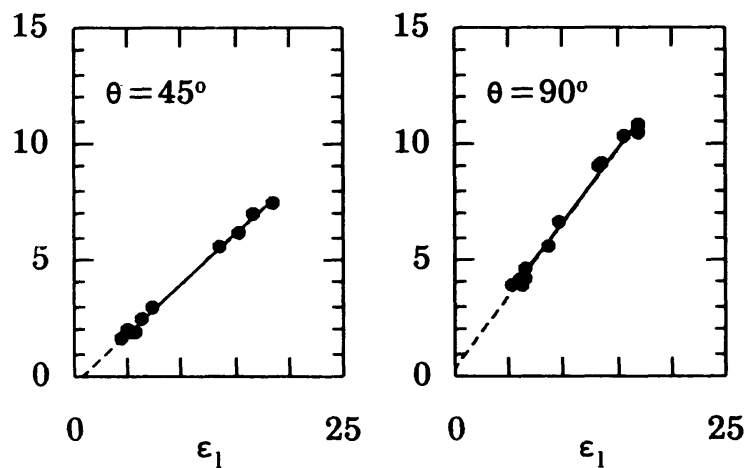

Figure 2 Examples of experimental $\varepsilon_{1}, \varepsilon_{w}$ data (in \%) obtained on (a) an IF1 steel, (b) an AKDQ steel and (c) a RIM steel. 
elongations and a narrow range of uniform plastic deformation) for 12 data points, compared to a common error of \pm 0.15 in the $r$ 's determined in the conventional manner. It is of interest that, for the cases where a non-zero $a$ intercept was obtained, the conventional and regression $r$ values differed by as much as 0.10 to 0.25 .

\section{b. Elasto-plastic transition of r-value}

Figure 3 shows the evolution of the conventional plastic $r$-value measured at various tensile strains (from 0.07 to 0.25 ) in three typical steels. The $r\left(\varepsilon_{l}\right)$ curve was derived in that range of deformation from the observed quasi-linear relationship between $\varepsilon_{1}$ and $\varepsilon_{w}$. It is obvious (from Eq. (9)) that the $a$ parameter, which was found to be generally positive in the case of inhomogeneous yielding and close to zero in most cases of homogeneous yielding, depends on the shape of the $r\left(\varepsilon_{1}\right)$ curve. The elastic $r$-value is also displayed, but it is clear that the elasto-plastic transition does not account for the variation in $r\left(\varepsilon_{1}\right)$ observed. As expected from Eqs (9) and (10), the conventional $r$ approaches the regression $r$ asymptotically at high strains.

The non-zero value of $a$ obtained even in the case of homogeneous yielding suggests that the $-\varepsilon_{w}$ vs $\varepsilon_{1}$ plots are curved between 0 and $15 \%$ longitudinal strain (as shown in Figure 1, they have to pass through the origin). This transition behaviour can be related to the rapid changes in grain orientation that take place as the $\gamma$-type fiber texture is partially destroyed and the texture evolves towards a stable configuration. Most of the measurements show positive $a$-value, which corresponds to a downward curvature of the first part of the $-\varepsilon_{q}$ vs $\varepsilon_{1}$ and is also associated with a decrease in $r$.

In most cases of inhomogeneous yielding, the conventional $r$-value decreases asymptotically with strain $(a>0)$ (Arthey et al., 1981; Hu, 1983; Lake et al., 1988). The question therefore arises about the effect of Lüders band propagation on the evolution of $r$-value during straining. The initial stages of plastic deformation, corresponding to Lüders band propagation, are in plane strain. After passage of the band, further deformation takes place by uniaxial tension. The first strain path involves lateral constraint and does not result from pure uniaxial loading; accordingly, two different $r$-values are displayed by the sample in the course of deformation. Lüders propagation leads to lengthening perpendicular to the front, which is generally inclined at $50^{\circ}$ to the tensile direction. Consequently $\varepsilon_{1}$ and $\varepsilon_{w}$ (positive) are nearly equal and $r_{\text {Lüders }}$ is $\sim-0.5$. Following propagation of the band, the rate of change of $r$ is most rapid during the first few percent of deformation (see the examples of the HSLA and AKDQ steels in Figure 3). The case of inhomogeneous yielding is shown schematically in Figure 4. Thus, as in homogeneous deformation, positive $a$ is seen to be associated with back extrapolation from a linear fit to data obtained at larger strains when $r$ is decreasing.

No clear quantitative relation can be derived between $r_{\mathrm{el}}$ and $r\left(\varepsilon_{1}\right)$ because of the complex phenomena involved in yielding and in the propagation of Lüders bands. Qualitatively, all the samples studied are represented by the three cases displayed in Figure 3. It appears that when $r_{\mathrm{pl}}$ tends to a value larger than $1.5, r_{\mathrm{el}}$ is systematically less than $r_{\mathrm{pl}}$ (Figure 3c). Figure 3a illustrates the case where $r_{\mathrm{pl}}$ is 
THE ELASTIC STRAIN RATIO
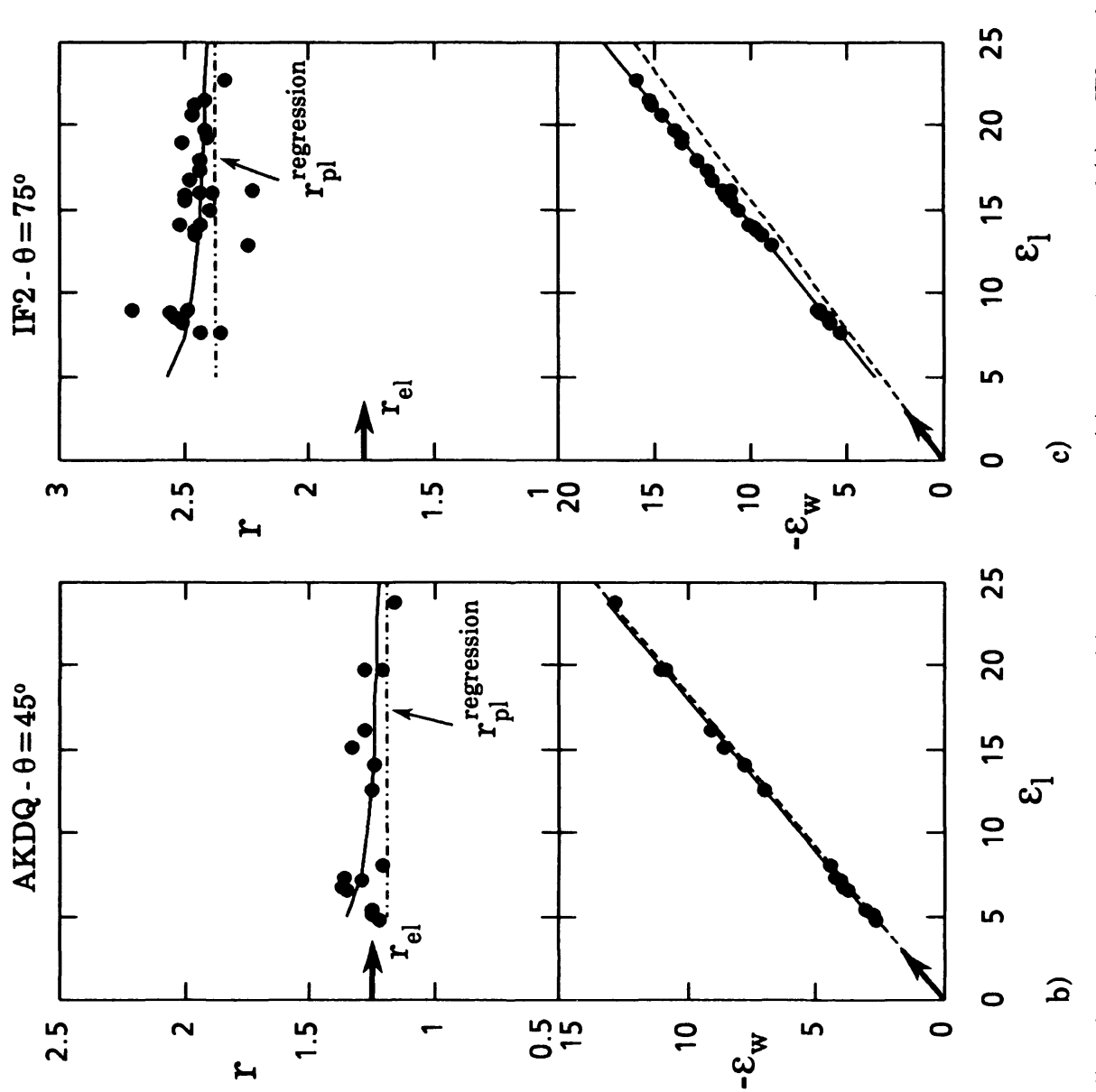

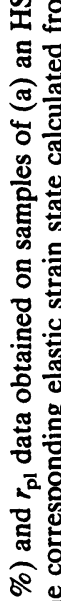

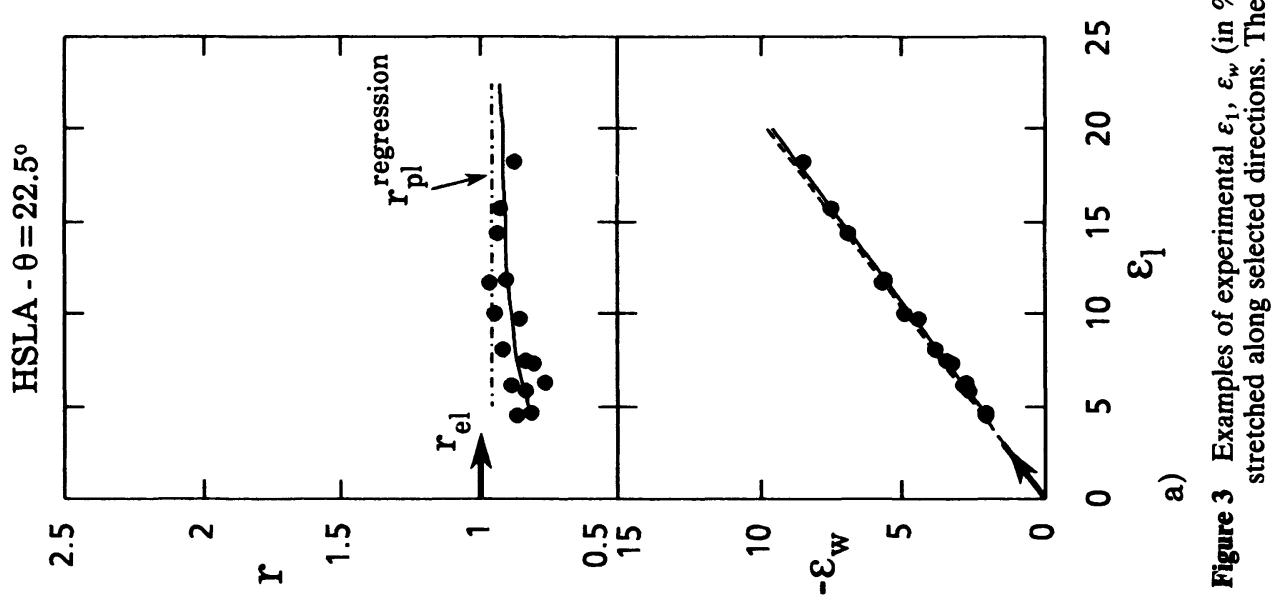




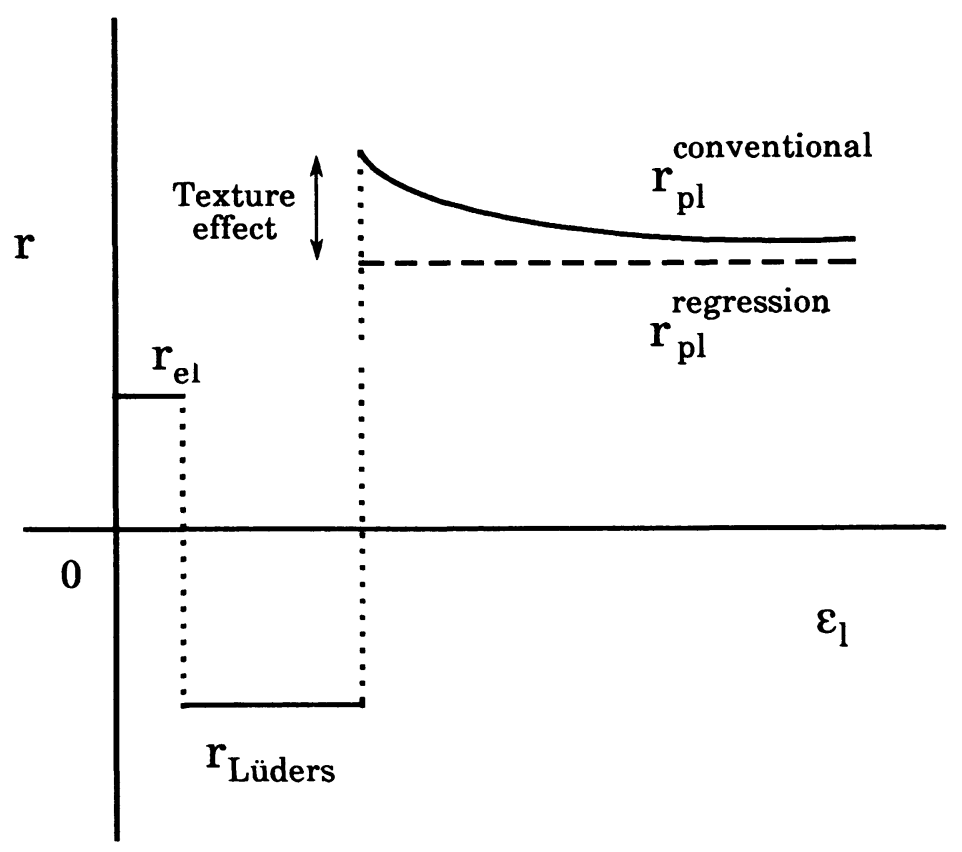

Figure 4 Schematic illustration of the evolution of $r$ in case of inhomogeneous yielding.

less than 1 and the corresponding $r_{\mathrm{el}}$ is generally greater than $r_{\mathrm{pl}}$. The elastic and plastic $r$ 's are approximately equal when $r_{p l}$ tends to a value between 1 and 1.5 (Figure 3b). These differences were not found to depend on the type of yielding behaviour.

\section{c. Planar variation of $r_{\mathrm{el}}$ and $r_{\mathrm{pl}}$}

In Figure 5, the angular variation of the elastic and plastic strain ratios are presented which pertain to the main ideal orientations observed experimentally in steel sheets. These textures were simulated using a gaussian type of orientation distribution so as to account for the normally occurring misorientation of individual grains with respect to an ideal orientation. A $15^{\circ}$ gaussian spread was employed. The $r_{\mathrm{pl}}(\theta)$ results were calculated using a relaxed constraint $\left(\mathrm{RC}_{3}\right)$ model, with bcc deformation taking place by mixed $\{110\}-\{112\}\langle 111\rangle$ glide with CRSS's on the two sets of systems such that $\tau_{\{112\}}=0.95 \tau_{\{110\}}$ (Daniel, 1990; Daniel and Jonas, 1990). Young's modulus data are also included since this quantity was used by Stickels and Mould (1970) to establish their empirical correlation with $r$-value. As expected, $E(\theta)$ and $r_{\mathrm{el}}(\theta)$ display opposite dependences on $\theta$. It is of interest that the $r_{\mathrm{el}}(\theta)$ curves follow the general tendencies displayed by the $r_{\mathrm{pl}}(\theta)$ curves, even though it was shown above that $r_{\mathrm{pl}}$ and $r_{\mathrm{el}}$ are not proportional along a given $\theta$ direction. Figure 5 also shows that $r_{\mathrm{el}}$ is always significantly less than $r_{\mathrm{pl}}$, except for components or specific directions along which $r_{p 1}$ is less than 1 . This can be related to the observations made above regarding Figure 3. 

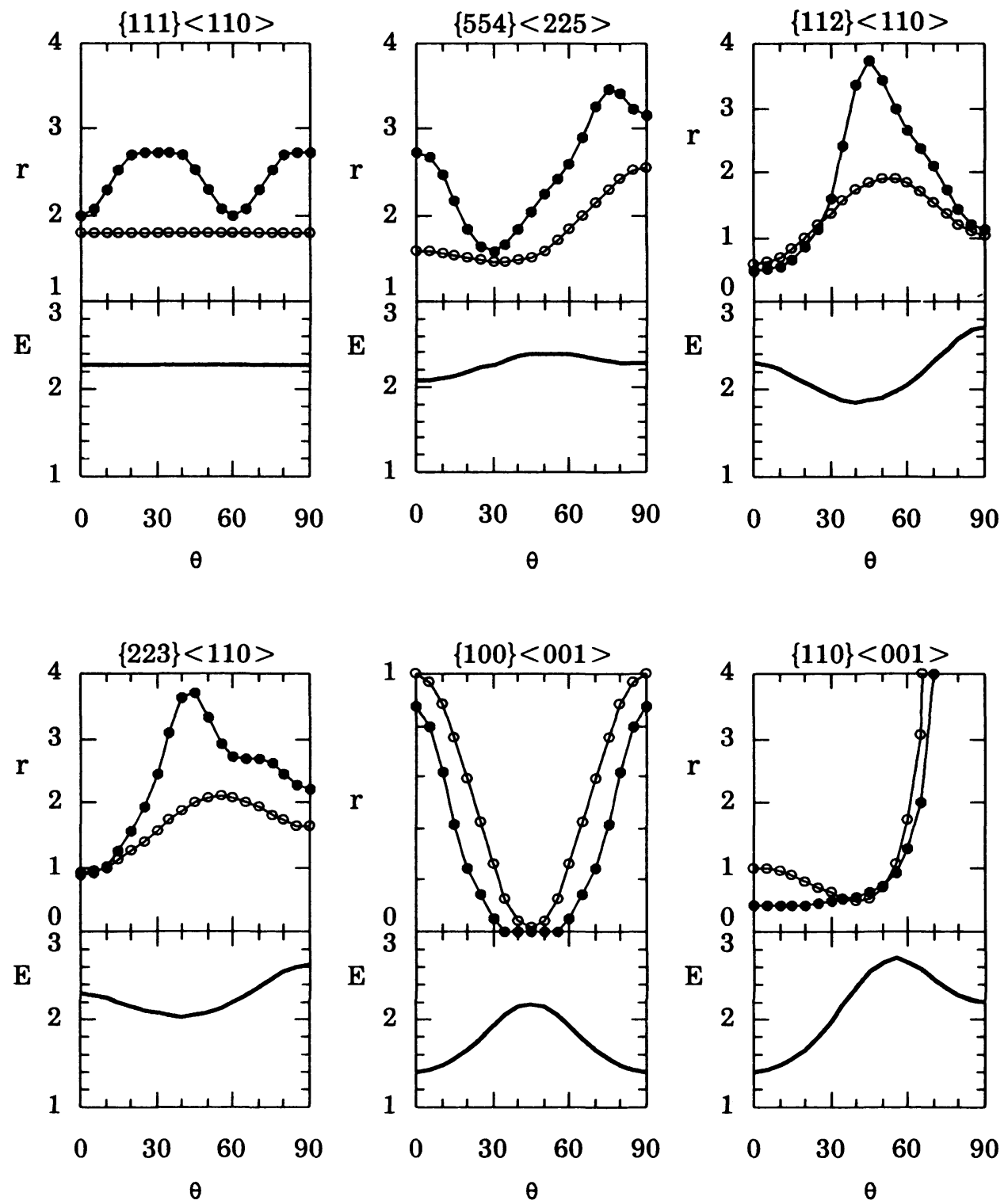

Figure 5 Predictions of $r_{\mathrm{pl}}(\theta)$ by the $\mathrm{RC}_{3}$ model $(O)$ and of $r_{\mathrm{el}}(\theta)$ by the Hill approximation $(O)$ for common texture components composed of a gaussian spread of $15^{\circ}$ around the respective ideal orientation. The dependence $E(\theta)$ is also indicated for comparison purposes.

Figure 6 compares $r_{\mathrm{el}}(\theta)$ and $r_{\mathrm{pl}}(\theta)$ for four of the steels studied. In the case of the low drawability steels (i.e. RIM and HSLA), the elastic and plastic strain ratios are in relatively good agreement. However, for the IF and AKDQ grades, the $r_{\mathrm{el}}(\theta)$ and $r_{\mathrm{pl}}(\theta)$ curves display similar tendencies, but without quantitative agreement. 

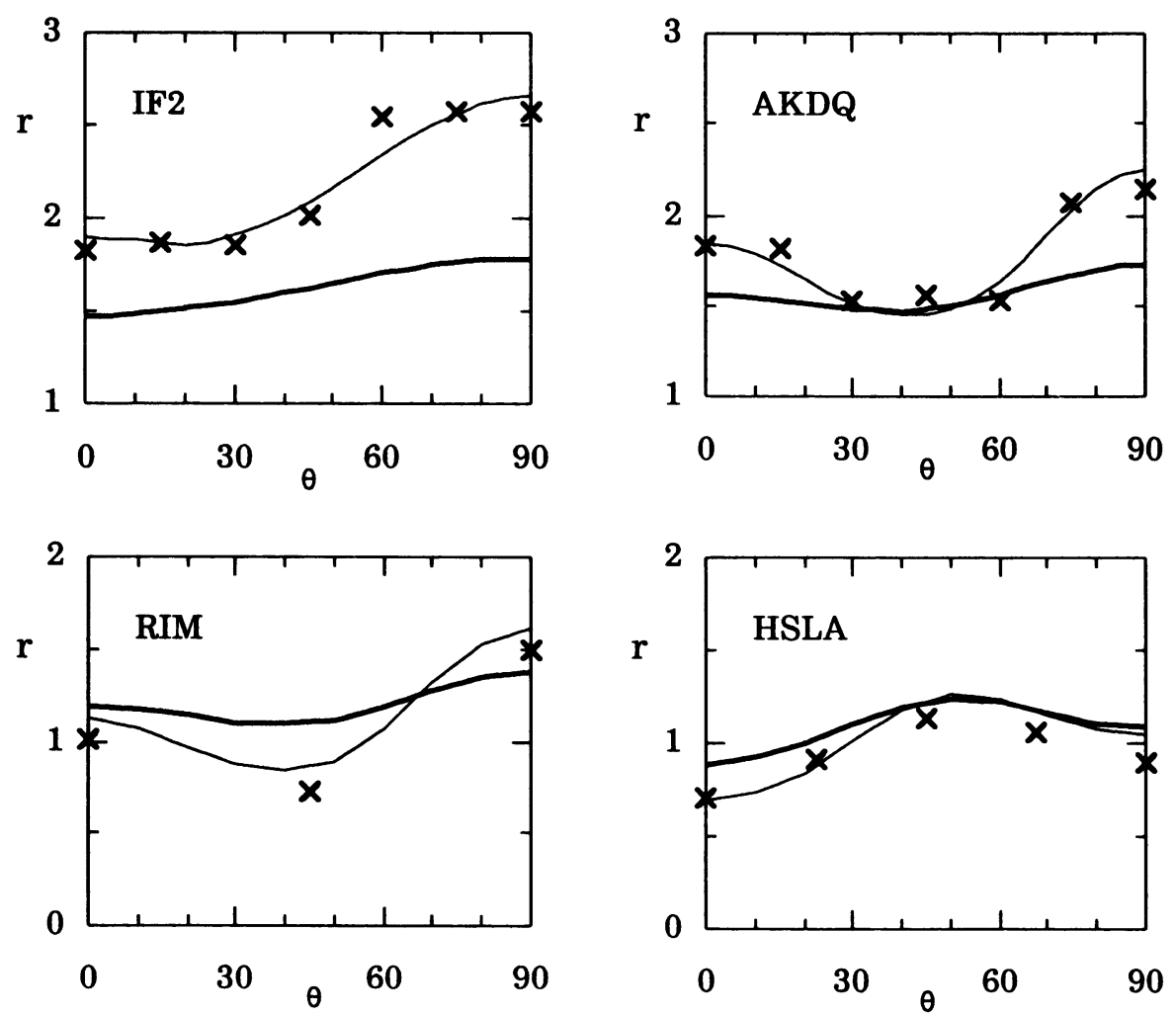

Figure 6 Comparison of $r_{\mathrm{pl}}(\theta)$ calculated from the $\mathrm{RC}_{3}$ model $(-)$ and $r_{\mathrm{el}}(\theta)$ determined from the Hill approximation $(-)$ with the experimental data $(x)$.

\section{d. Empirical correlations between $r_{\mathrm{el}}$ and $r_{\mathrm{pl}}$}

The results described above suggested that it would be of interest to derive empirical correlations between the anisotropies of the elastic and plastic $r$-values. Such analyses were carried out in the past using Young's modulus (Stickels and Mould, 1970) or the ultrasonic velocities (Bussière et al., 1987). However, it appears that $r_{\mathrm{el}}$ is physically more relevant since it characterizes the resistance to thinning in the elastic range of deformation much as $r_{p l}$ typifies it for the plastic range.

The empirical correlations between the values of elastic and plastic $\bar{r}, \Delta r$ and $\delta r(=r(90)-r(0))$ are illustrated in Figure 7. ( $\delta r$ represents the tendency to form two-fold ears.) No detailed regression analysis was carried out due to the limited number of samples. Qualitatively, it can be concluded that the dispersion of the data is within the experimental error $\left( \pm 0.1\right.$ in $\left.r_{\mathrm{pl}}\right)$, indicating that the elastic strain ratio, readily measured ultrasonically (i.e. non-destructively), can be used to estimate the plastic one with the same accuracy as in the Stickels and Mould analysis. 

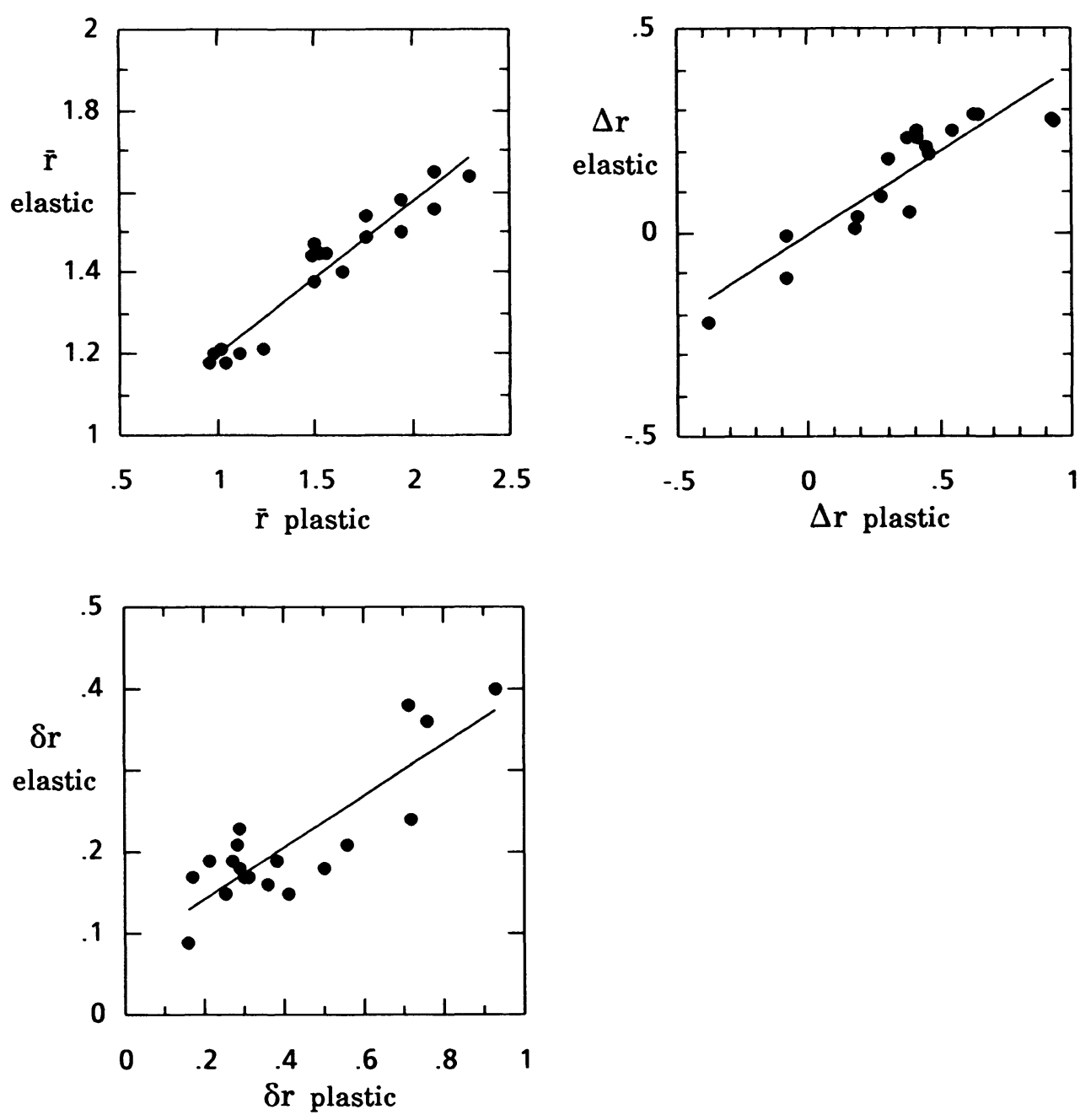

Figure 7 Correlation between the elastic and plastic $\bar{r}, \Delta r$ and $\delta r$. The plastic quantities are experimental data and the elastic ones were calculated from ultrasonic data.

\section{CONCLUSIONS}

Analysis of the evolution of $r_{\mathrm{pl}}$ during conventional tensile deformation shows that the changes in $r_{\mathrm{pl}}$ are not related to the elastic-to-plastic transition. Nor is Lüders band propagation responsible for the increase or decrease in $r_{\mathrm{pl}}$ observed. It appears instead that the variations in $r_{\mathrm{pl}}$ are due to texture evolution, which is in turn responsible for non-zero values of $a$ in the case of homogeneous deformation.

As a result of the rapid initial evolution of $r_{\mathrm{pl}}$, the back-extrapolated (texture-related) value is probably higher than either the regression or the 
conventional value when $a>0$ (and $r_{\mathrm{pl}}$ is decreasing), and lower when $a<0$ (and $r_{\mathrm{pl}}$ is increasing). For plastic $r$ 's greater than $1.5, r_{\mathrm{el}}$ is systematically less than $r_{\mathrm{pl}}$; this difference decreases when the plastic $r$ is less than 1.5, and becomes negative when $r_{\mathrm{pl}}<1$.

This study shows that the elastic strain ratio can be readily determined from ultrasonic measurements. The $r_{\mathrm{el}}(\theta)$ and $r_{\mathrm{pl}}(\theta)$ curves display similar tendencies. So, the elastic $r$, determined nondestructively, can be used to estimate its plastic counterpart with the aid of empirical relationships. Such correlations are physically better based than the ones relating $r$-value to Young's modulus or ultrasonic velocity.

\section{ACKNOWLEDGEMENTS}

The authors are indebted to Prof. J. Szpunar of McGill University for assisting with the texture measurements. They are grateful to the Kawasaki Steel Corporation, Stelco Steel Inc., Dofasco Inc. and the Algoma Steel Corporation for supplying steel sheets. They acknowledge with gratitude the financial support received from the Canadian Steel Industry Research Association, the Natural Sciences and Engineering Research Council of Canada, and the Ministry of Education of Quebec (FCAR program).

\section{References}

Arthey, R. P. and Hutchinson, W. B. (1981). Metall. Trans., 12A, p. 1817.

Bunge, H. J. (1974). Kristall Tech., 9, p. 413.

Bunge, H. J. (1982). Texture Analysis in Materials Science, Butterworths, London.

Bussière, J. F., Jen, C. K., Makarow, I., Bacroix, B., Lequeu, Ph. and Jonas, J. J. (1987). Proc. 2nd Int. Symp. on Nondestructive Characterization of Materials, Montreal, July 21-23, 1986, Ed. by J. F. Bussière et al., Plenum Press, New York, p. 523.

Daniel, D. (1990). PhD Thesis, McGill University, Montreal, Canada.

Daniel, D. and Jonas, J. J. (1990). Metall. Trans., 21A, p. 331.

Daniel, D., Sakata, K. and Jonas, J. J. (1991). Trans. ISIJ, 31, p. 696.

Hill, R. (1948). Proc. Roy. Soc. London, Ser. A, 193, p. 281.

Hirao, M., Hara, N. and Fukuoka, H. (1988). J. Acoust. Soc. Am., 84, p. 667.

Hirao, M., Fukuoka, H., Fujisawa, K. and Murayama, R. (1989). Metall. Trans., 20A, p. 2385.

Hu, H. (1983). Metall. Trans., 14A, p. 1521.

Lake, J. S. H., Willis, D. J. and Fleming, H. G. (1988). Metall. Trans., 19A, p. 2805.

Lankford, W. T., Snyder, S. C. and Bauscher, J. A. (1950). Trans. ASM, 42, p. 1197.

Liu, Y. C. (1983). Metall. Trans., 14A, p. 1199.

Liu, Y. C. and Johnson, L. K. (1985). Metall. Trans., 16A, p. 1531.

Sakata, K., Daniel, D., Jonas, J. J. and Bussière, J. F. (1990). Metall. Trans., 21A, p. 697.

Sayers, C. M. (1982). J. Phys., D15, p. 2157.

Stickels, C. A. and Mould, P. R. (1970). Metall. Trans., 1A, p. 1303.

Thompson, R. B., Lee, S. S. and Smith, J. F. (1987). Ultrasonics, 25, p. 133.

Thompson, R. B., Smith, J. F., Lee, S. S. and Johnson, G. C. (1989). Metall. Trans., 20A, p. 2431.

Welch, P. I., Ratke, L. and Bunge, H. J. (1983). Z. Metallkde, 74, p. 233.

Whiteley, R. L., Wise, D. E. and Blickwede, D. J. (1961). Sheet Met. Ind., 38, p. 349. 